\title{
Network Modelling and Visualisation Analysis of the Undergraduate Dental Curriculum System in China
}

\author{
Qian Ren1, Xiao Pang2, Xiaoyu Liu', Qiao Zheng1, Ting He³, Die Hu³, Xuelian Jiang3, \\ Linglin Zhang1,3* \\ ${ }^{1}$ Education Affairs Office, West China Hospital of Stomatology, Sichuan University, Chengdu, China \\ ${ }^{2}$ College of Computer Science, Sichuan University, Chengdu, China \\ ${ }^{3}$ State Key Laboratory of Oral Diseases, National Clinical Research Centre for Oral Diseases, Department of Cariology and \\ Endodontics, West China Hospital of Stomatology, Sichuan University, Chengdu, China \\ Email: ${ }^{\star}$ zhll_sc@163.com
}

How to cite this paper: Ren, Q., Pang, X., Liu, X.Y., Zheng, Q., He, T., Hu, D., Jiang, X.L. and Zhang, L.L. (2021) Network Modelling and Visualisation Analysis of the Undergraduate Dental Curriculum System in China. Journal of Computer and Communications, 9, 38-51.

https://doi.org/10.4236/jcc.2021.96003

Received: May 6, 2021

Accepted: June 15, 2021

Published: June 18, 2021

Copyright $\odot 2021$ by author(s) and Scientific Research Publishing Inc. This work is licensed under the Creative Commons Attribution International License (CC BY 4.0).

http://creativecommons.org/licenses/by/4.0/

\section{(c) (i) Open Access}

\begin{abstract}
Objectives: This study aims to present the characteristics of the undergraduate dental curriculum system using network modelling and visualisation analysis based on complex network theory, thus providing a theoretical foundation for the course development and curriculum reform. Methods: The correlation coefficient was used to quantify the intensity of the correlation between courses, and a visualisation complex network of the dental curriculum was built to explore the curriculum pattern from a dynamic perspective. Further, the statistical measurements of curriculum network were adopted to express the most relevant topological features. Subsequently, the minimum spanning tree and parallel coordinates plot were drawn to explore the curriculum community structure, quantify the key courses, and present different courses in time and space relationships. Results: The correlation analysis results show that the courses are closely related to each other. The main courses focus on pathology, pathophysiology, oral anatomy and physiology, closely connecting almost all medicine-related courses. The whole course network has an average degree value of 41.53 , and a clustering coefficient of 0.78 , indicating an obvious small-world characteristic. From the perspective of curriculum attributes, the number of public and theoretical courses was more than that of skills and practical courses. Moreover, the academic performance of skills and practical courses was lower than that of public and theoretical courses. Conclusion: The undergraduate dental courses have a progressive structure from basic professional knowledge to professional skills, which is reasonable for the dental education in China. However, some efforts towards curriculum reform based on this study are needed.
\end{abstract}




\section{Keywords}

Complex Network, Dental Education, Curriculum, Data Visualisation, Educational Data Mining

\section{Introduction}

In 1907, Canadian missionary Dr. Lindsay established the Ren Ji Dental Clinic, which introduced modern dentistry in China and laid the foundation of dental education in the nation. After more than 100 years of practice and development, China has formed a distinctive dental education model [1]. The goal of undergraduate dental education is to produce safe, competent, and ethical practitioners equipped with the necessary knowledge, communication skills, professionalism, and behaviour appropriate for the independent practice of dentistry. Based on the curriculum requirements of China's dental undergraduate education standards, the teaching system can be divided into three stages-basic medicine, clinical medicine, and stomatology matched with corresponding courses [2]. The huge and complex dental curriculum system contains extensive information revealing the links between courses and the rules of course development [3]. By excavating the educational data about curriculum, the internal connections and underlying laws can be uncovered, which not only provides some decision-making information for course creation but also inspire curriculum optimisation.

Data Mining, popularly known as Knowledge Discovery in Databases (KDD), is the automated or convenient extraction of patterns representing knowledge implicitly stored in large databases [4] [5] [6] [7] [8]. By means of computer technology, the huge data can be visualized into a complex network composed of nodes connected together in a specific way for internal connection analysis. In the network, nodes represent different objects as per the meaning assigned to them (such as users, courses, etc.), and the edges between nodes indicate some kind of connection between objects [9] [10]. Based on complex network theory, the quantitative and topological analysis of educational data mining can effectively and reasonably represent the interaction and correlation degree of many components in a complex system, thus revealing the potential relationship between different variables. In recent years, network modelling and visualisation analysis of big data using complex networks has been given great importance in various fields [10] [11]. Till date, such analysis has led to a wealth of innovative results in education in areas such as course recommendation, grade prediction, and online personalised education [3] [12] [13] [14].

Focusing on college education, researchers had used several visualisation measures based on complex network to excavate the data of college education. The office of Digital Learning at MIT and Harvard Research Committee at Harvard University used two years' data of 68 open online courses offered on edX to 
explore trends span. They found that the overall participation in their online courses remained substantial, and the average growth has been steady [15]. Seering provided a visualisation tool mapping the relationships among learning outcomes in the process of developing subject content [16]. In 2014, Goh et al. used the complex network method to construct networks of statistically significant transitions between variable pairs from a high-dimensional and multiscale dataset of teaching practices observed in Grade 5 and Grade 9 mathematics classes. From the Minimum Spanning Trees (MST) and Planar Maximally Filtered Graphs (PMFG) of the transition networks, they established that teaching knowledge as truth and teacher-dominated talking serve as hubs for teaching practices in Singapore and determined the complex teacher-student interaction teaching practice sequence [17]. To visualise the hidden structure in an academic curriculum, Aldrich advanced the prerequisite network and found that individual courses served different roles in the organisation, such as information sources, hubs, and bridges. Moreover, the curriculum prerequisite network represents the intrinsic, hard-wired constraints on the flow of information in a curriculum and is the organisational context within which learning occurs [18].

The dental undergraduate curriculum system is a huge and complex system in which courses are interwoven into a network. Using complex network mapping, the curriculum system may provide a better visualisation on understanding and development of curriculum pattern in undergraduate dental education. The present study intends to analyse the correlation between undergraduate dental courses, build a curriculum network dynamically, and explore its internal structural characteristics and setting rules, thus providing guidance for further curriculum setting and reform. This is the first study to conduct network modelling and visualisation analysis for undergraduate courses in a dental school, and could serve as a "roadmap" to guide administrators and faculty as they build and revise a curriculum, and for students as they try to navigate the curriculum.

\section{Methods}

\subsection{Data Acquisition and Filtering}

This study was approved by West China Hospital of Stomatology, Sichuan University. The courses were required at the undergraduate level and graded according to the percentage system. Further, the scores of students whose school status has changed were excluded. Finally, a total of 78 subjects were included and divided into 10 categories (Supporting Information Table 1) of coursesEnglish Courses, Physical Education Courses, Ideological and Political Courses, Comprehensive Literacy Courses, Medical Foundation Courses, Medical Practice

Table 1. Descriptive statistics of the undergraduate dental curriculum network.

\begin{tabular}{ccccc}
\hline & The degree & The betweenness & The clustering coefficient & The mean path \\
\hline The max value & 62 & 124.26 & 0.94 & null \\
The average value & 41.53 & 20.54 & 0.78 & 1.52
\end{tabular}


Courses, Introduction to Stomatology, Dental Foundation Courses, Dental Clinical Courses, and Dental Practice Courses. The performance data of 134 five-year undergraduates graduated in 2019 were used to model the complex network.

\subsection{Pearson Correlation Coefficient Analysis}

The Pearson correlation coefficient was used to quantify the intensity of the correlation between courses, thus depicting the key courses and curriculum structure. Further, the original performance data were standardised to exclude the influence of students' efforts and the level of intelligence on the correlation between courses. The original correlation coefficient and the "standardized" correlation coefficient were compared and analysed to show the consistency and complementarity of the two types of the correlation coefficient. Based on the Pearson correlation coefficient analysis, the matrix heat map was drawn to describe the strength of the relationship between courses [10] [19].

\subsection{Dynamic Threshold Network}

The force-oriented model was used to lay out the node link graph, which was imagined as a physical system. The courses were regarded as nodes, and subsequently, the straight line between nodes was defined as the strength of the correlation relationship. With the repulsive force, all nodes moved under the interaction force. Further, when the system reaches equilibrium, the optimal layout is obtained. By increasing the threshold, only the edges not less than the threshold were retained in the network, and users could dynamically change the threshold to observe the network structure with different degrees of simplification [20].

\subsection{Measurements of Complex Network}

The complex network has specific topological features that characterise connectivity. Therefore, the discrimination and analysis of complex networks rely on the use of measurements that can express the most relevant topological features and their subsequent classification [21]. The measurements used in this study are described in the following subsection.

\subsubsection{The Degree and Degree Distribution}

The degree refers to the number of nodes associated with the nodes to be studied, which is an important characteristic of a vertex. To quantify the key courses of stomatology, the degree values of each node in the course network were calculated, as well as the average degree $k_{\text {avg }}=\frac{1}{N} \sum_{i=1}^{N} k_{i}$, where $k_{i}$ is the degree of node $i$. Subsequently, the degree distribution function was fitted to analyse the structural characteristics of the curriculum network [10] [21].

\subsubsection{The Clustering Coefficient}

Similar to degree, this clustering coefficient is also an important characteristic for network analysis [21] [22]. The clustering coefficient is usually used to indicate the interconnectedness of a complex network. The clustering coefficient for 
vertex $i$ is: $C^{i}=\frac{N_{\Delta}(i)}{N_{3}(i)}$, where $N(i)$ is the number of triangles involving vertex $i$, and $N_{3}(i)$ is the number of connected triples with $i$ as the central vertex. Based on the clustering coefficient for each vertex, the clustering coefficient for a network can be defined as follows: $\bar{C}=\frac{1}{N} \sum_{i} C_{i}$.

\subsubsection{The Betweenness Centrality}

The betweenness is another popular index, measuring the extent to which a node lies on the shortest paths between all the other nodes in a graph [23]. The betweenness of each node and the average betweenness of the course network were calculated to reveal the courses that played the role of connecting the past and the future in the course network. The betweenness calculation formula of any node is as follows: $\delta_{i j}(t)=\frac{\partial_{i j}(t)}{\partial_{i j}}$, where $\partial_{i j}$ is the number of shortest paths between node $v_{i}$ and node $v_{j}$, and $\partial_{i j}(t)$ is the number of shortest paths through node $v_{i}$ among all shortest paths between node $v_{i}$ and node $v_{j}$.

\subsubsection{The Average Path}

The average path is an important parameter to measure the connectivity and accessibility of a complex network [18]. In the network, the distance between two nodes is defined as the shortest path, and the average distance of all node pairs is the average path of the network. In the network of undergraduate courses of stomatology, the shortest path between any two courses was obtained, and the average path of the network was calculated. The formula is as follows: $L=\frac{2}{n(n-1)} \sum_{i, j \in V} d_{i j}$.

\subsection{Minimum Spanning Tree}

In order to highlight the key structure of the course network, the graph structure of the course is simplified as the minimum spanning tree [11]. For the minimum spanning tree, set $G^{\prime}=\left\{V^{\prime}, E^{\prime}\right\}, V^{\prime}=\{0,1,2, \cdots N\}$ represents the course set, $E^{\prime}=\left\{e_{i j}, i, j \in V^{\prime}\right\}$ contains $N-1$ edges, connects each node in $V^{\prime}$, and minimizes the sum of edge weights, which are the correlation coefficients between courses.

\subsection{The Parallel Coordinates Plot}

The parallel coordinates plot is a visualisation method for analysis with multiple properties [24] [25]. In this study, the class program number, time, category, and average score were combined, and deviation data were standardised into a high-dimensional space. To explore the characteristics of points in the high-dimensional space, each dimension was reflected as a vertical axis, parallel and equidistantly arranged on the horizontal axis. A high-dimensional point was represented as a polyline of inflection points on these parallel vertical axes, and 
the position on the $K_{\mathrm{th}}$ vertical axis indicates the value of this point in the $K_{\mathrm{th}}$ dimension.

\subsection{Data Analysis}

A descriptive analysis of data was carried out.

\section{Results}

\subsection{Pearson Correlation Coefficient Analysis}

Correlation coefficients have been calculated for each pair of courses and are presented as a heat map in Figure 1. Based on the course category order, Figure 1(a)

(a)

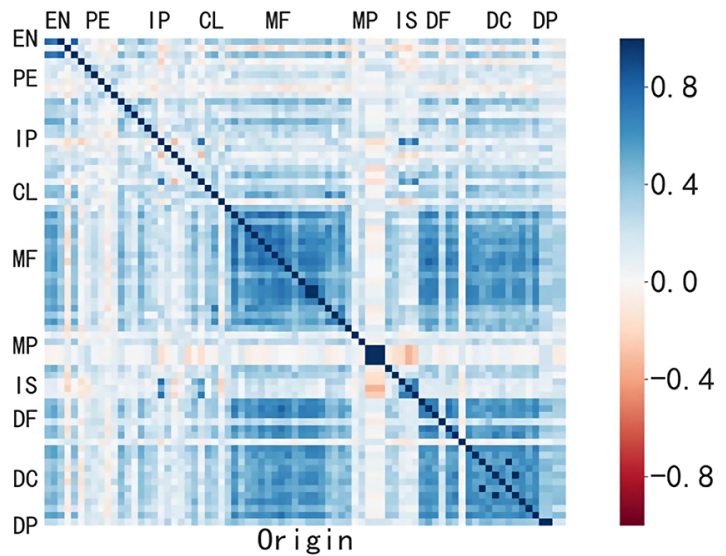

(b)

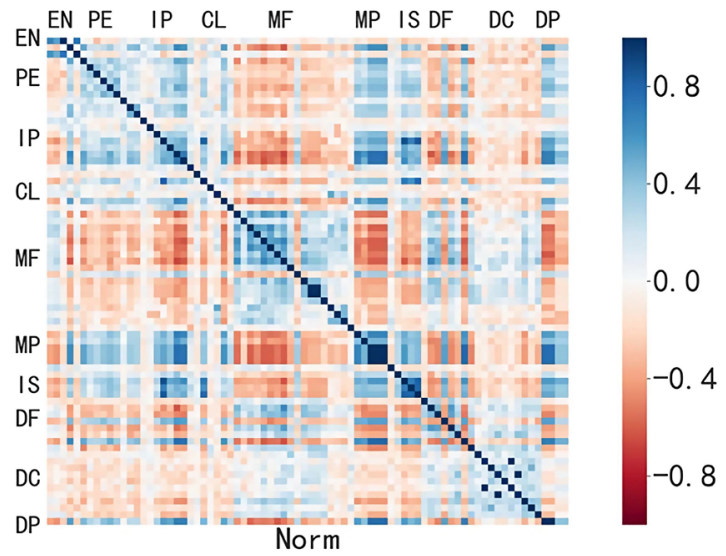

Figure 1. The matrix heat map of pairwise course correlation. (a) the original correlations; (b) the normalised correlations. The correlation value of each pair of courses is represented by the colour of the corresponding cell: higher correlation values refer to blue and lower values to red. Courses are ordered by category, which, in turn, are the 10 categories according to the curriculum program: English Courses (EN), Physical Education Courses (PE), Ideological and Political Courses (IP), Comprehensive Literacy Courses (CL), Medical Foundation Courses (MF), Medical Practice Courses (MP), Introduction to Stomatology (IS), Dental Foundation Courses (DF), Dental Clinical Courses (DC), and Dental Practice Courses (DP). 
and Figure 1(b) depict the original and "standardised" visual matrix heat maps of correlation coefficient, respectively. The colour of the small squares reflects the level of correlation between courses. As shown in Figure 1, the correlation coefficient near the diagonal of the matrix heat map is relatively high. Overall, the courses are closely related to each other, either within or between categories, and the original correlation coefficient is larger than the "standardised" correlation coefficient.

\subsection{Dynamic Threshold Network}

Figure 2 shows the curriculum network diagram with four different thresholds in the dynamic process. The node colour represents its category, and the numbers on the node are the abbreviations of the corresponding course. When the threshold value is 0.4, Physical Education, Situation and Policy, and Oral English courses are stripped out, while the rest of the curriculum remains tightly connected. When the threshold value is 0.5 , the Introduction to Stomatology and practice courses of stomatology, including clinical skill experiments and clinical practice, are separated and formed into independent groups. When the threshold value is 0.6 , the clinical medicine curriculum is further stripped out, and the rest of the courses are stomatology specialisations, which are closely related to each other. When the threshold value is 0.7 , only a few courses are still connected,

(a)

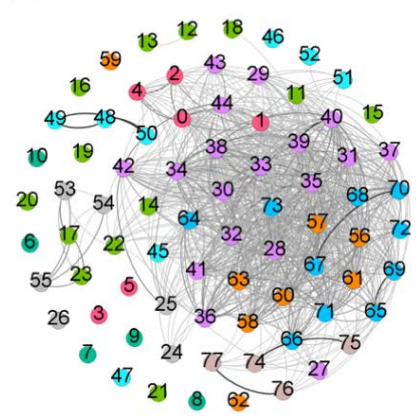

(c)

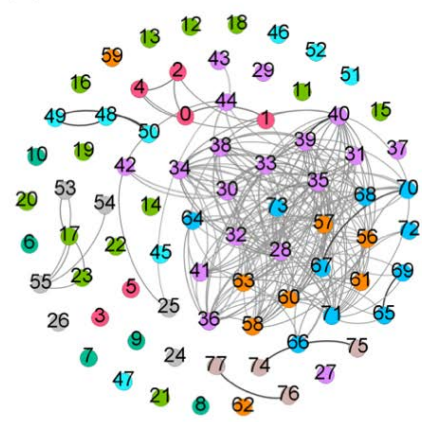

(b)

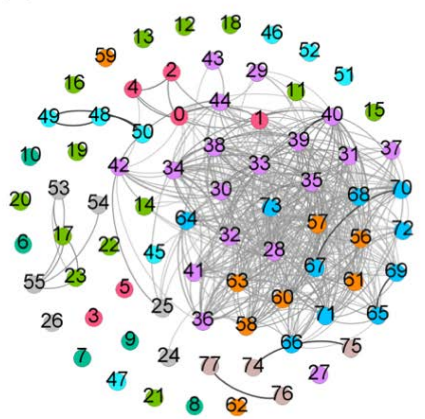

(d)

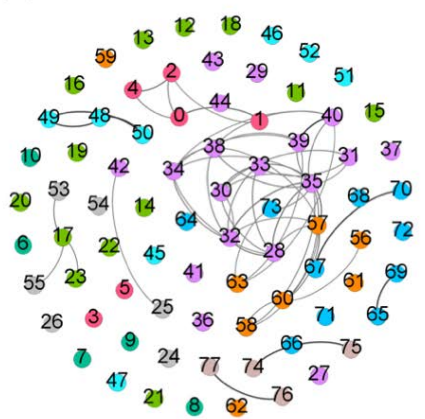

Figure 2. Curriculum networks with different connection thresholds. Colours of the nodes represent its category according to the cultivation program. Course networks for four typical threshold $K$ values are represented in (a)-(d). (a) $K=0.4$, (b) $K=0.5$, (c) $K=$ 0.6 , (d) $K=0.7$. 
including Oral Histopathology, Oral Anatomy and Physiology, Oral Biology, Oral materials Science, Medical Microbiology, Pathology, Pharmacology, Physiology, and Biochemistry, all of which are basic oral or medical courses.

\subsection{Description of Statistical Characteristics}

The statistical results of complex network in Table 1 showed that the courses with large degree were primarily medical foundation and dental clinical courses. The maximum degree value of nodes in the course network was 62, while the average degree value was 41.53 . There were 51 nodes with values greater than the average degree value. The courses with small degree value were primarily physical education and ideological and political courses. By fitting the degree distribution function, it is similar to the Poisson distribution, as shown in Figure 3. The average betweenness value of the whole course network is 20.54 . In addition, the average clustering coefficient of the whole course network was 0.78 , and the average path was 1.52 .

\subsection{The Minimum Spanning Tree}

As shown in Figure 4, the minimum spanning tree of 78 undergraduate dental courses has a clear and compact structure. The node colour represents the course category, and the node size is proportional to the degree. The curriculum structure model has a remarkable "trunk-branch" structure. As shown in Figure 4 , the main courses are highly consistent with the key course groups obtained by dynamic threshold network analysis. The main courses focus on Pathology, Pathophysiology, Oral Anatomy and Physiology, and Oral Biology, closely connecting almost all medical-related courses. At the branch of the curriculum network, there are clear categories of general education courses, such as English, Physical Education, and Ideological and Political courses. In addition, it is

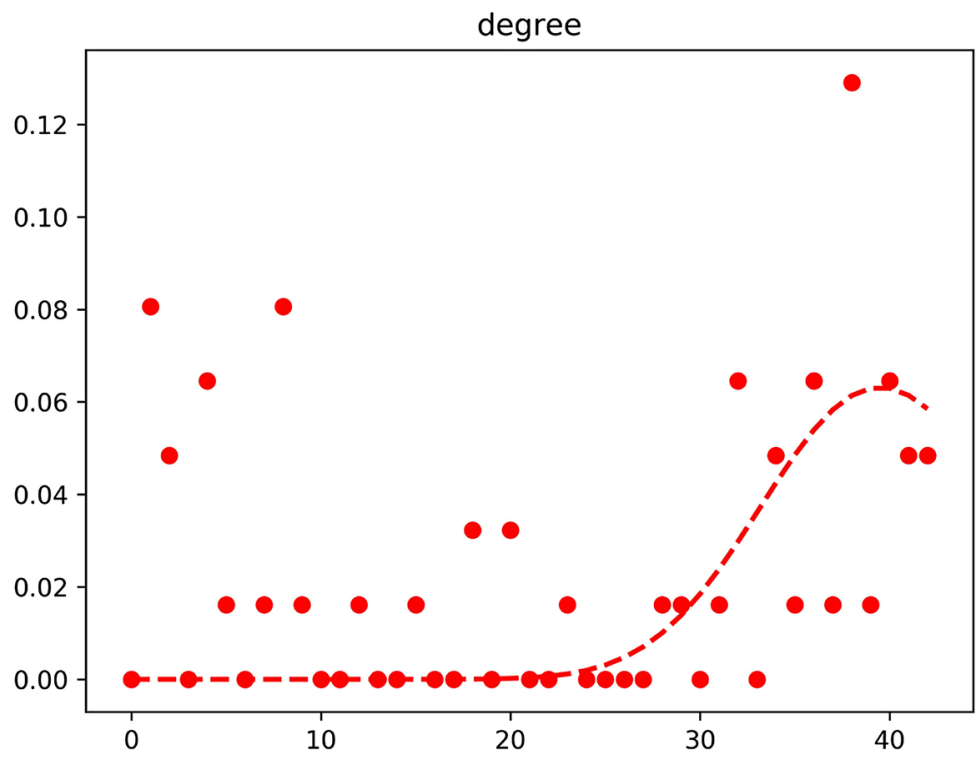

Figure 3. The degree distribution of the undergraduate dental curriculum network. 


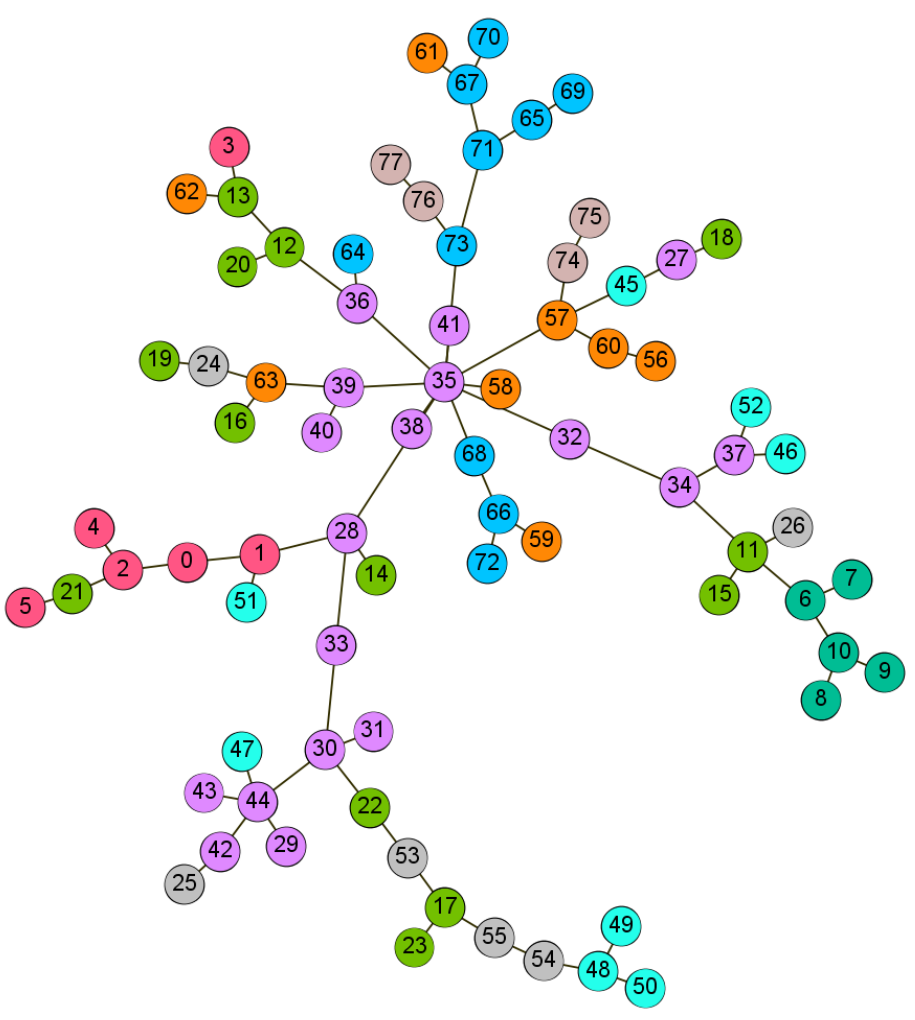

Figure 4. The minimum spanning tree of the curriculum network. The number of nodes represents different courses, and the different colours represent categories.

not difficult to find that some experimental courses are connected to corresponding theoretical courses.

\subsection{The Parallel Coordinates Plot}

The parallel coordinate visualisation analysis of undergraduate dental courses is shown in Figure 5. Except for the internship of stomatology in the fifth year, the number of courses in other semesters were evenly distributed. In terms of time dimension, the course presents a harmonious progressive relationship. From the public, medical, and dental courses, a gradual learning process with Chinese characteristics has been formed for the dental education, which is quite different from the cultivation of foreign professionals in stomatology. From the perspective of curriculum attributes, the number of public and theoretical courses was obviously more than that of skills and practical courses. Moreover, the academic performance of skills and practical courses was lower than that of public and theoretical courses.

\section{Discussion}

Learning is a complex cognitive process that can be influenced by the structure of an academic curriculum. Students' performance data usually reflect whether the curriculum is scientific or reasonable and may provide new ideas for curriculum exploration. In this study, the undergraduate dental curriculum was treated 

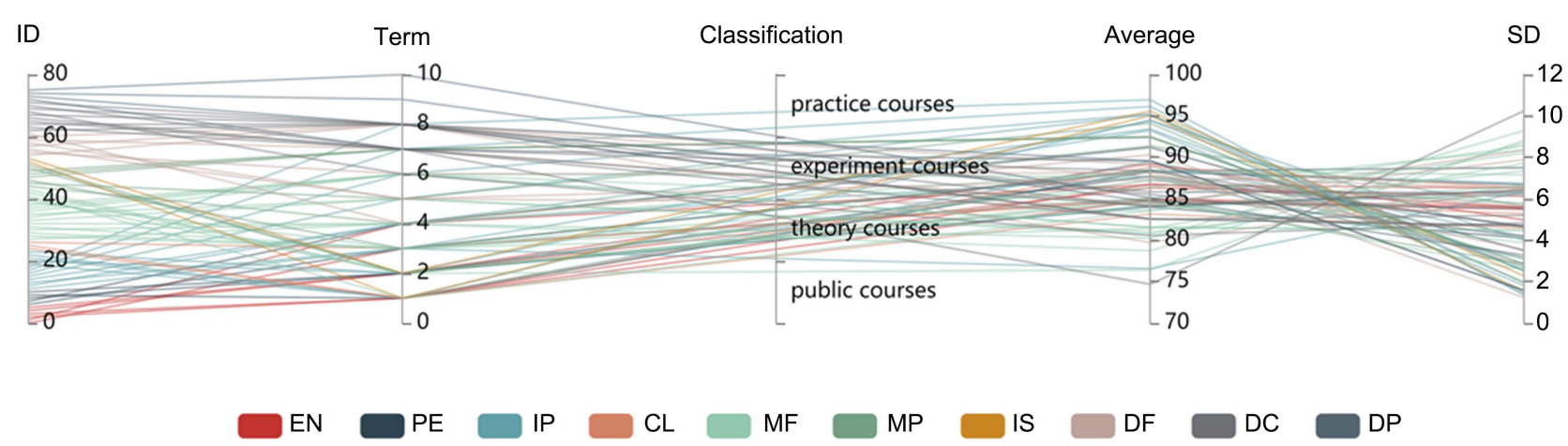

Figure 5. The parallel coordinates plot of the undergraduate dental curriculum network. Colours of the lines represent its category according to the cultivation program.

as a complex system, and student scores were used to conduct the network modelling and visual analysis.

In the Pearson correlation coefficient analysis, the course indexes are arranged consecutively according to the courses of the same category, which can reduce the visual confusion and allow clearer observation of the correlation between courses of different categories. As shown in Figure 1, the courses are closely related to each other, either within or between categories. It's worth noting that the correlation coefficient near the diagonal is relatively high, indicating that the courses of the same category have a high similarity, especially with a high internal correlation. However, the overall original correlation coefficient is larger than the "standardised" correlation coefficient in value, which may be because there is a certain correlation between students' comprehensive quality and course scores.

Through the computer visual simulation technology, the complex relationships, such as the strength of the correlation between courses and their priorities, can be visualised in a dynamic form so that the key courses can be dynamically presented. In the dynamic threshold network, the courses were treated as nodes in a complex network, and the strength of the correlation was defined as the distance between nodes. By increasing the threshold of distance between courses, the network structure of courses evolves from full connectivity to isolated points. As shown in Figure 2, with the increase of the threshold value, the courses start to be separated from the fully connected network, and the edge and key courses can be quickly identified. In the process of dynamic visualisation analysis, Oral English, Physical Education, Politics, and other courses were gradually separated, and the dental courses were always closely related, which reflected their core position in the curriculum network. There is a close relationship between the courses of stomatology and clinical medicine, which indicates the rationality of clinical medicine as a pre-curriculum. Notably, with the increasing threshold, practice courses are stripped out earlier than basic medical courses both in clinical medicine and dental courses, which may indicate that there is a problem of emphasizing theory over practice in the undergraduate teaching of stomatology. In general, the undergraduate courses of stomatology have a progressive course 
structure from basic professional knowledge to skill training. Most of the undergraduate courses of stomatology have a high correlation, while a few of the public courses have a low correlation. Given this scenario, it is necessary to further strengthen the learning of high relevance node courses in teaching and pay attention to the combination of theory with practice.

As the undergraduate dental curriculum system is a complex network, its degree, betweenness, clustering coefficient, and the average path can be used to describe the topological characteristics. The degree refers to the number of nodes associated with the node to be studied, reflecting the status and importance of the course in the whole course system [10] [21]. Betweenness centrality speaks to the broader-scale travers' ability of a network and the role played by individual nodes in that level of connectivity [23]. In the course network, the combination of node degree and betweenness can be used as a more comprehensive index to measure the importance of course nodes and excavate the key courses in the teaching of stomatology. In the present study, it was not difficult to find that nodes with larger degree values also tend to have higher betweenness values. Similarly, using the statistical characteristic of clustering coefficient and the average path, the problem of setting up teaching content can be revealed, which is of positive significance for promoting the rationalisation and scientific quality of teaching content arrangement [22]. The whole course network had a large clustering coefficient and a small average path, indicating an obvious small-world characteristic. The topological measurements of the course network related to each other closely, and suggest that courses with high degree or betweenness value should be studied as the focus of the major, which will help to strengthen the stability of learners' knowledge network.

In the minimum spanning tree, the undergraduate courses of stomatology have a progressive course structure from basic professional knowledge to professional skills, and some experimental courses are connected to corresponding theoretical courses. Interestingly, the curriculum system also presents a harmonious and progressive relationship in the time dimension, as shown in the parallel coordinates plot. However, students in skills and practical courses did not learn as well as those in public and basic courses. This may be related to the curriculum setting. For example, in our school, the undergraduates take public and medicine foundation courses in the first two years. During the third year and the first term of the fourth year, medicine practice courses comprise majority of courses, and students have the opportunity to rotate between several clinical departments such as Otolaryngology and Endocrinology. At this phase, dental foundation courses, including Oral Histology and Pathology, and Oral Anatomy and Physiology are taught. In the second term of the fourth year, students focus on the dental clinical and experimental courses. In the final year, all students complete the clinical practice in the hospital of stomatology. It can be seen that the dental courses are compacted in the last two years. It is not easy to engage in extensive and deep study with such a tight schedule. Thus, there is a need for curricular reform that includes greater integration and coherence, and efforts 
have also been taken in China [26] [27]. To leave a preliminary impression of dentistry on students at an early stage, some schools adjusted the curriculum and set courses related to dentistry, such as Introduction to Stomatology and Exploratory Learning in Stomatology. These courses are more effective at encouraging students to study and develop comprehensively. However, current reforms are far from sufficient, and more efforts need to be taken.

As curriculum reform is generally done without a clear view of the shape of the existing curriculum, this study may provide a solution to this problem by rendering important aspects of the overall curriculum visible and subject to quantitative, systems-level analysis. As shown in the curriculum network, when courses were differently labelled with category-specific colours, it became evident that there was strong integration across topics within a category but less integration between categories. A strategy for controlling curricular integration is through the management of bridge courses, which can carry a considerable load of the information traffic between course categories. Further, managing knowledge communities is another method of curricular integration. A curriculum might be integrative yet ineffective at delivering a learning experience. Coherence should also be a goal so that curricula make sense to the students that navigate them. Furthermore, it is our goal to cultivate expert professionals with profound cultural heritage, solid professional foundation, strong sense of innovation, and broad international vision. As dental students, they must be trained to excel and innovate in the clinical practice of stomatology in the future. Therefore, the cultivation of students' professional knowledge and skills of stomatology based on competence should be strengthened except their comprehensive quality.

\section{Conclusion}

Based on complex network theory, network modelling and visualisation analysis were conducted to depict the characteristics of the undergraduate dental curriculum system. Most undergraduate courses of stomatology have a high correlation, and the whole course network had a large clustering coefficient, a small average path, indicating an obvious small-world characteristic. Moreover, the undergraduate courses of stomatology have a progressive course structure from basic professional knowledge to professional skills. In brief, this study quantitatively analysed the academic performance of dental undergraduates, characterised the curriculum structure, dynamically and intuitively presented the key courses, and provided a theoretical foundation for course setting and curriculum reform. However, some efforts for curriculum reform based on this study are needed.

\section{Acknowledgements}

Thanks the Education Affairs Office, West China Hospital of Stomatology, Sichuan University for giving permission to access the departmental database. 
And this work was supported by Soft Science Research Project of West China Hospital of Stomatology, Sichuan University.

\section{Conflicts of Interest}

The authors declare no conflicts of interest regarding the publication of this paper.

\section{References}

[1] Wu, Z.Y., Zhang, Z., Jiang, X.Q. and Guo, L. (2010) Comparison of Dental Education and Professional Development between Mainland China and North America. European Journal of Dental Education, 14, 106-112. https://doi.org/10.1111/j.1600-0579.2009.00599.x

[2] Ling, J. and Fu, Y. (2007) Recent Changes in the Curriculum of Chinese Dental Schools. Journal of Dental Education, 71, 1447-1456. https://doi.org/10.1002/j.0022-0337.2007.71.11.tb04416.x

[3] Chen, X. and Xue, C. (2018) Network Visual Exploration for the Cooperation Map of Courses in Different Major Curricula. Educational Sciences. Theory \& Practice, 18, 2874-2884.

[4] Han, J. and Kamber, M. (2006) Data Mining: Concepts and Techniques. In: Data Mining Concepts Models Methods \& Algorithms, Vol. 5, 2nd Edition, 1-18.

[5] Azuaje, F., Witten, I.H. and Frank, E. (2006) Data Mining: Practical Machine Learning Tools and Techniques 2nd Edition. BioMedical Engineering OnLine, 5, Article No. 51. https://doi.org/10.1186/1475-925X-5-51

[6] Al-Razgan, M., Al-Khalifa, A.S. and Al-Khalifa, H.S. (2014) Educational Data Mining: A Systematic Review of the Published Literature 2006-2013. In: Herawan, T., Deris, M. and Abawajy, J., Eds., Proceedings of the First International Conference on Advanced Data and Information Engineering, Springer, Singapore, 711-719. https://doi.org/10.1007/978-981-4585-18-7_80

[7] Mudasir, A., Majid, Z. and Muheet, A. (2020) An Intelligent Prediction System for Educational Data Mining Based on Ensemble and Filtering Approaches. Procedia Computer Science, 167, 1471-1483. https://doi.org/10.1016/j.procs.2020.03.358

[8] Shyamala, K. and Rajagopalan, S.P. (2006) Data Mining Model for a Better Higher Educational System. Information Technology Journal, 5, 198-203. https://doi.org/10.3923/itj.2006.560.564

[9] Amaral, L.A.N. and Ottino, J.M. (2004) Complex Networks. The European Physical Journal B, 38, 147-162. https://doi.org/10.1140/epjb/e2004-00110-5

[10] Tang, J., Jiang, B., Chang, C.C. and Luo, B. (2012) Graph Structure Analysis Based on Complex Network. Digital Signal Processing, 22, 713-725. https://doi.org/10.1016/j.dsp.2012.04.011

[11] Ortega, G.J., Sola, R.G. and Jesús, P. (2008) Complex Network Analysis of Human ECoG Data. Neuroscience Letters, 447, 129-133. https://doi.org/10.1016/j.neulet.2008.09.080

[12] Méndez, G., Ochoa, X. and Chiluiza, K. (2014) Techniques for Data-Driven Curriculum Analysis. Proceedings of the 4th International Conference on Learning Analytics and Knowledge, Indianapolis, 24-28 March 2014, 148-157. https://doi.org/10.1145/2567574.2567591

[13] Zou, Q., Liu, D., Duan, H. and Xing, L. (2016) An Evaluation Model of Academic 
Record of Electronic and Information Engineering Base on Complex Network Theory. 2016 4th International Conference on Management, Education, Information and Control, Shenyang, 24-26 September 2016, 554-558.

https://doi.org/10.2991/meici-16.2016.116

[14] Gajewski, L.G., Choloniewski, J. and Holyst, J.A. (2016) Key Courses of Academic Curriculum Uncovered by Data Mining of Students' Grades. Acta Physica Polonica $A$, 129, 1071-1076. https://doi.org/10.12693/APhysPolA.129.1071

[15] Dean, H.A., Isaac, C., Justin, R., Austun, C.C., Jacob, W., Northcutt, C.G., et al. (2015) HarvardX and MITx: Two Years of Open Online Courses Fall 2012-Summer 2014. SSRN Electronic Journal. https://doi.org/10.2139/ssrn.2586847

[16] Seering, J., Huang, L. and Willcox, K. (2015) Mapping Outcomes in an Undergraduate Aerospace Engineering Program. 12th American Society for Engineering Education Annual Conference \& Exposition, Seattle, Washington, 14-17 June 2015.

[17] Goh, W.P., Kwek, D., Hogan, D. and Cheong, S.A. (2014) Complex Network Analysis of Teaching Practices. EPJ Data Science, 3, Article No. 36.

https://doi.org/10.1140/epjds/s13688-014-0034-9

[18] Aldrich, P.R. (2015) The Curriculum Prerequisite Network: Modeling the Curriculum as a Complex System. Biochemistry and Molecular Biology Education, 43, 168-180. https://doi.org/10.1002/bmb.20861

[19] Reggiani, E., D’Arnese, E., Purgato, A. and Santambrogio, M.D. (2017) Pearson Correlation Coefficient Acceleration for Modeling and Mapping of Neural Interconnections. 2017 IEEE International Parallel and Distributed Processing Symposium Workshops, Lake Buena, 29 May-2 June 2017, 223-228.

https://doi.org/10.1109/IPDPSW.2017.63

[20] Geva, A.B. and Peled, A. (2000) Simulation of Cognitive Disturbances by a Dynamic Threshold Semantic Neural Network. Journal of the International Neuropsychological Society, 6, 608-619. https://doi.org/10.1017/S1355617700655108

[21] Costa, L.F., Rodrigues, F.A., Travieso, G. and Villas, P.R. (2007) Characterization of Complex Networks: A Survey of Measurements. Advances in Physics, 56,167-242. https://doi.org/10.1080/00018730601170527

[22] Zhang, J., Yang, C., Jin, Z. and Li, J. (2018) Dynamics Analysis of SIR Epidemic Model with Correlation Coefficients and Clustering Coefficient in Networks. Journal of Theoretical Biology, 449, 1-13. https://doi.org/10.1016/j.jtbi.2018.04.007

[23] Brandes, U. (2001) A Faster Algorithm for Betweenness Centrality. The Journal of Mathematical Sociology, 25, 163-177. https://doi.org/10.1080/0022250X.2001.9990249

[24] Gennady, A. and Natalia, A. (2001) Constructing Parallel Coordinates Plot for Problem Solving. International Symposium on Smart Graphics 2001, Hawthorne, 21-23 March 2001, 9-14.

[25] Sand, C., Lechler, T., Schuh, P. and Franke, J. (2018) Potentials for Error Detection and Process Visualization in Assembly Lines Using a Parallel Coordinates Plot. Applied Mechanics and Materials, 882, 10-16. https://doi.org/10.4028/www.scientific.net/AMM.882.10

[26] Sun, W., Hu, Q., Zhang, H., Liu, Y., Bensch, B., Wang, W., et al. (2011) A New Dental Curriculum for Chinese Research Universities. Journal of Dental Education, 75, 1374-1382. https://doi.org/10.1002/j.0022-0337.2011.75.10.tb05184.x

[27] Wang, Y.H., Zhao, Q. and Tan, Z. (2016) Current Differences in Dental Education between Chinese and Western Models. European Journal of Dental Education, 21, e43-e49. https://doi.org/10.1111/eje.12216 